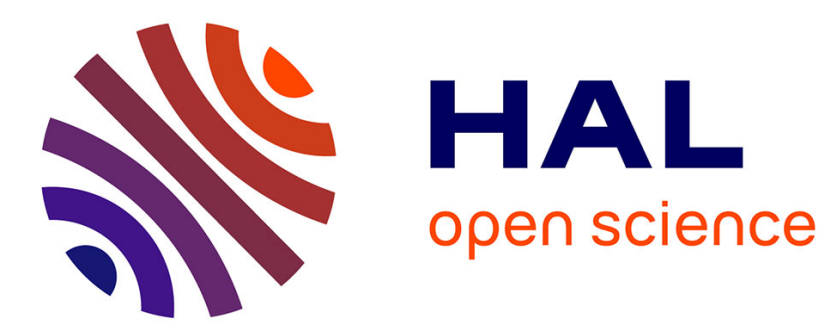

\title{
Thermal and magnetic properties of gadolinium by acoustic detection of optically and magnetically induced temperature oscillations
}

\author{
C. Glorieux, J. Thoen
}

\section{- To cite this version:}

C. Glorieux, J. Thoen. Thermal and magnetic properties of gadolinium by acoustic detection of optically and magnetically induced temperature oscillations. Journal de Physique IV Proceedings, 1994, 04 (C7), pp.C7-271-C7-274. 10.1051/jp4:1994764 • jpa-00253293

HAL Id: jpa-00253293

https://hal.science/jpa-00253293

Submitted on 1 Jan 1994

HAL is a multi-disciplinary open access archive for the deposit and dissemination of scientific research documents, whether they are published or not. The documents may come from teaching and research institutions in France or abroad, or from public or private research centers.
L'archive ouverte pluridisciplinaire HAL, est destinée au dépôt et à la diffusion de documents scientifiques de niveau recherche, publiés ou non, émanant des établissements d'enseignement et de recherche français ou étrangers, des laboratoires publics ou privés. 


\title{
Thermal and magnetic properties of gadolinium by acoustic detection of optically and magnetically induced temperature oscillations
}

C. Glorieux and J. Thoen

\section{Laboratorium voor Akoestiek en Warmtegeleiding, Departement Natuurkunde, Katholieke Universiteit} Leuven, Celestijnenlaan 200D, 3001 Leuven, Belgium

\begin{abstract}
Periodically intensity modulated optical excitation and magnetical heat dissipation has been used to produce temperature oscillations in three gadolinium samples with differences in the quality of crystallinity. Depending on the circumstances the acoustically detected temperature signal can contain information on specific heat capacity, thermal conductivity or magnetic susceptibility of the material. We have investigated the magnetic field and temperature dependence of these physical properties in the neighbourhood of the critical point $\left(\mathrm{T}=\mathrm{T}_{\mathrm{c}}\right.$ and $\left.\mathrm{B}=0\right)$.
\end{abstract}

\section{INTRODUCTION}

Gadolinium is a rare earth metal which is magnetic because of the presence of 7 unpaired electrons in the 4f orbital. At the Curie point which is close to room temperature a transition occurs from the paramagnetic to the ferromagnetic state. From a theoretical point of view, a prediction of the critical behavior of $\mathrm{Gd}$ is rather ambiguous. On the one hand the $4 \mathrm{f}$ orbital is half full, thus isotropic, suggesting that Gd should belong to the Heisenberg universality class. On the other hand its hexagonal crystal symmetry, which via the spin-orbit coupling has a major influence on the spin-spin interactions, is anisotropic, which could indicate Ising behavior. From high resolution specific heat capacity results obtained by ac calorimetry, Bednarz et al. [1] find that the critical behavior of $\mathrm{Gd}$ is not at all simple; i.e. there is a crossover behavior between different regimes approaching the Curie temperature.

We have used acoustic detection of temperature oscillations to investigate the temperature and field dependence of the thermal and magnetic properties of three Gd samples of different quality, one was the high quality sample investigated by Bednarz et al.

\section{EXPERIMENTAL SETUP AND ANALYSIS}

\subsection{Photoacoustic detection}

When a sample is periodically illuminated $\left(I=I_{0} \exp (i \omega t)\right)$, its temperature distribution can be calculated solving the heat conduction equation which is inhomogeneous due to the heat source term $\left(I_{0} \beta / 2 \kappa\right) \exp (i \omega t+\beta x)$, with $\beta$ the optical absorption coefficient and $\kappa$ the thermal conductivity. In a gas- 
sample-gas configuration, assuming $\beta^{-1}<\mu$ ( $\mu=$ thermal diffusion length) and a small gas-sample effusivity ratio $e_{g} / e_{s}$ leads to a complex temperature oscillation [2] :

$$
\theta_{0} \exp (i \omega t)=\frac{I_{0}(1-R)}{\sqrt{2 \omega}(1+i) e_{s}} \frac{\exp (\sigma l)+\exp (-\sigma l)}{\exp (\sigma l)-\exp (-\sigma l)} \exp (i \omega t)
$$

with $R$ the sample reflectivity, $\sigma=(1+\mathrm{i})(\omega / 2 \alpha)^{1 / 2}=(1+\mathrm{i}) / \mu, 1$ the sample thickness and $\alpha$ the sample diffusivity. In the case of 'low' frequencies the heat wave reaches the back of the sample $(\mu \approx 1)$, and both sample thermal diffusivity and effusivity can be determined simultaneously by iteratively inverting expression (1). Since the photoacoustic signal is proportional to $\theta_{0}$, using proper calibration procedures, the sample thermal diffusivity and effusivity can be determined from the amplitude and phase of the photoacoustic signal. If the sample density is known, then also specific heat capacity and thermal conductivity can be determined.

\subsection{Magnetically induced temperature oscillations}

When a magnetic sample is placed in a varying magnetic field $B$, then its magnetization $M$ will change, and the energy increase $(\mathrm{B} \uparrow)$ or decrease $(\mathrm{B} \downarrow)$ of the spins will cause an energy flow to or from the lattice, equal to $\mathrm{B}$ times the magnetization change. Provided $\mathrm{B}$ is uniform over the sample, the spins then act as a uniform heating or cooling source in the sample. When the external field is amplitude modulated $\left(B=B_{0}+B_{1} \exp (i \omega t)\right)$ the dissipated power equals :

$$
P_{m}=\frac{d}{d t} \int_{0}^{M} B d M=\frac{d}{d M} \int_{0}^{M} B d M \cdot \frac{d M}{d B} \frac{d B}{d t}
$$

Using the relation $\mathrm{dM} / \mathrm{dB}=\chi$, for the magnetic susceptibility, and inserting the time dependence of $\mathrm{B}$ gives :

$$
\begin{aligned}
P_{m} & =i \omega \chi B_{1}\left(B_{0}+B_{1} \exp (i \omega t)\right) \exp (i \omega t) \\
& =i \omega B_{0} B_{1} \chi \exp (i \omega t)+i \omega \chi B_{1}{ }^{2} \exp (2 i \omega t) \\
& =P_{m, \omega}+P_{m, 2 \omega}
\end{aligned}
$$

In addition to an $\omega$ component, the magnetic power also contains a $2 \omega$ component. Except for $T=T_{c}$ $(\chi \rightarrow \infty)$, and if $\mathrm{B}_{1}<<\mathrm{B}_{0}$ and if $\mathrm{d} \chi / \mathrm{dB}$ is not too large, we can Taylor expand the susceptibility $\chi(\mathrm{B})$ to first order and omit higher order terms :

$$
\chi\left(B_{0}+B_{1} \exp (i \omega t)\right)=\chi\left(B_{0}\right)+\left.\frac{d}{d B}\right|_{B_{0}} \cdot B_{1} \exp (i \omega t)
$$

The $\omega$ component of the magnetic power will thus have a $\omega$ as well as a $2 \omega$ component :

$$
\begin{gathered}
P_{m, \omega}=B_{0} \chi\left(B_{0}\right) i \omega B_{1} \exp (i \omega t), \\
P_{m, 2 \omega}=\left[\chi\left(B_{0}\right)+\left.B_{0} \frac{d \chi}{d B}\right|_{B_{0}}\right] i \omega B_{1}^{2} \exp (2 i \omega t) .
\end{gathered}
$$


Performing a similar calculation as described in 2.1. for a gas-sample-gas configuration and replacing the heat source term in the thermal diffusion equation by the uniformally distributed magnetic power term $\mathbf{P}_{\mathbf{m}}$ ' $\dot{\kappa}$, we find for the 'magnetoacoustic' front surface temperature oscillation :

$$
\theta_{0} \exp (i \omega t)=P_{m} \exp (i \omega t) /(i \omega \rho C)
$$

with $\rho$ and $C$ the density and specific heat capacity of the sample.

From the magnetoacoustic $\omega$ signal, using (5) and (7) and inserting the specific heat capacity obtained from the photoacoustic measurements, we can determine the field and temperature dependence of the magnetic susceptibility $\chi(B, T)$. Combining (6) and (7) for $\mathrm{B}_{0}=0$ one can determine $\chi(T, B=0)$ from the $2 \omega$ signal.

\section{RESULTS AND DISCUSSION}

We have investigated three gadolinium samples : sample I $(220 \mu \mathrm{m})$ is commercially pure polycrystalline $\mathrm{Gd}$, sample II $(365 \mu \mathrm{m})$ is $99.99 \%$ pure crystalline $\mathrm{Gd}$ and sample III $(220 \mu \mathrm{m})$ has highly improved quality, by performing a sequence of annealing cycles, and was obtained from Bednarz et al.[1], who have used it for their experiments.
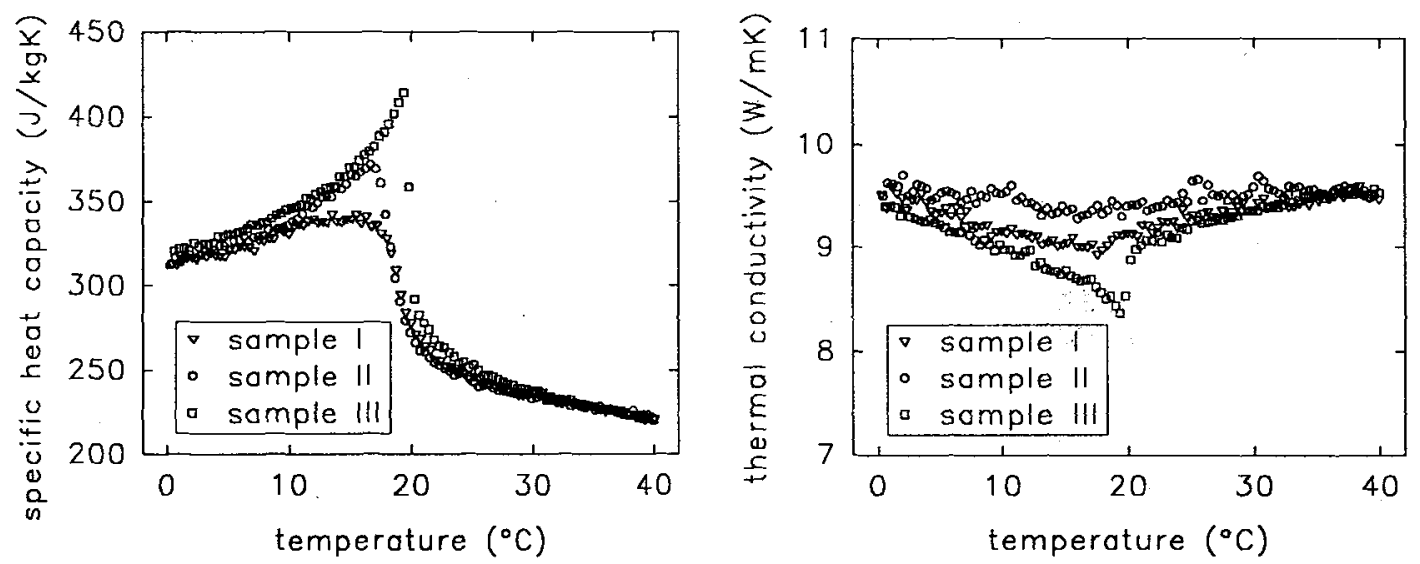

Fig. I: Photoacoustic data for the specific heat and thermal conductivity of sample I, II and III near $T_{c}(B=0)$

The left graph of Fig. 1 shows the influence of the quality of the samples on the specific heat capacity, obtained from photoacoustic data. A decreasing sample quality causes $T_{c}$ to decrease and yields smoother curves. Our specific heat capacity result for sample III in zero magnetic field was within the experimental error equal to the ac calorimetry data of Bednarz et al. [1]. We also carried out a series of temperature scans of sample III in the presence of a constant magnetic field with a chosen set of values. With increasing field strength the system moves away from the critical state resulting in less pronounced specific heat capacity anomalies. A detailed analysis of the resulting $C\left(B_{0}, T\right)$ results, in particular for the critical isotherm will be given elsewhere [3]. For sample I we have also determined the specific heat capacity by (uniformally) electrically and periodically heating the sample in a gas-sample-gas configuration, and find the same temperature dependence as for the photoacoustic experiment.

For all of the three samples, the thermal conductivity shows a dip at $T_{c}$, which is sharper with better crystallinity, and smoothes in a magnetic field. This clearly indicates an influence of the magnetic spins on the phonon-phonon interactions, possibly via spin-lattice relaxation like mechanisms. 

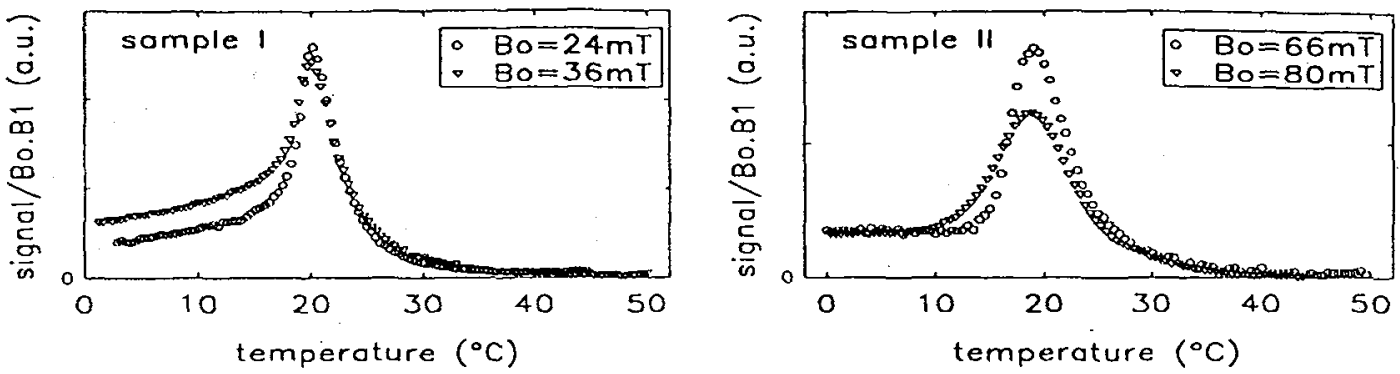

Fig.2: Temperature dependence of the magnetoacoustic $\omega$ signal, divided by $B_{0} B_{I}$ for sample I and II in the presence of different magnetic fields
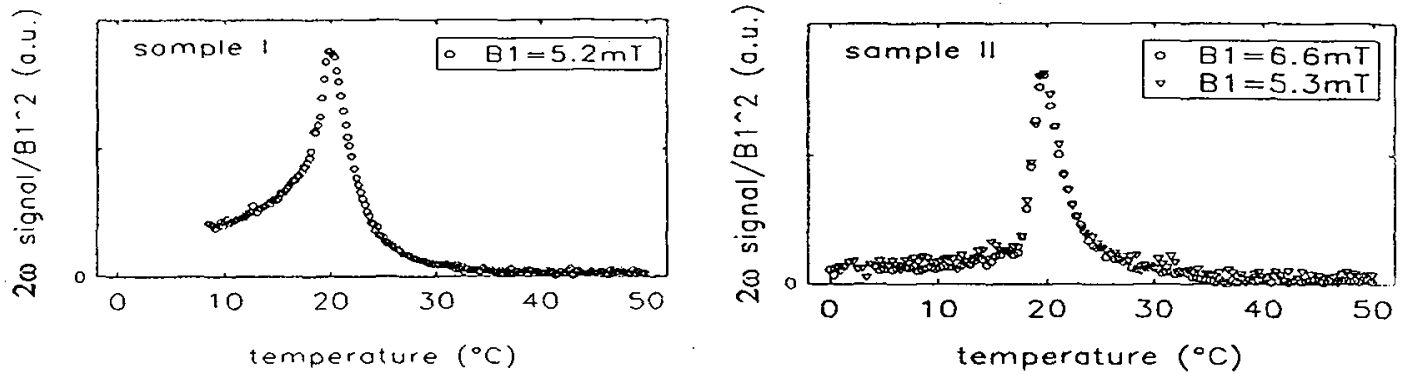

Fig. 3: Temperature dependence of the magnetoacoustic $2 \omega$ signal divided by $B_{I}^{2}$ for sample I and II in zero static field

Fig. 2 shows the field dependence of the $\omega$ component of the magnetoacoustic signal divided by the product of the magnetic fields $B_{0} B_{1}$. According to expressions (5) and (7), this quantity is proportional to the susceptibility. Results are shown for sample I and II. It is expected that a declining crystal quality causes a $\mathrm{T}_{\mathrm{c}}$ distribution over the different microscopic crystals and domains, resulting in a smoother transition. Therefore, the susceptibility peak is more rounded for the polycrystalline sample I compared to sample II (single crystal). An increasing magnetic field also results in smoothing the susceptibility peak : the susceptibility anomaly is smeared out when moving away from the critical point $\left(T=T_{c}, B_{0}=0\right)$.

It is clear from expressions (5) and (6) that for $\mathrm{B}_{0}=0$, the magnetoacoustic $\omega$ signal is zero, and the $2 \omega$ signal is proportional to the zero-field magnetic susceptibility. The proportionality between the $2 \omega$ signal and $B_{1}^{2}$ is confirmed by the results in Fig.3. The 20 signal divided by $B_{1}^{2}$ is the same for two different values of $B_{I}$. The temperature dependence of the signal is comparable to the data of Stetter et al. [4], although he studied the influence of annealing circumstances on the magnetic susceptibility of thin film Gd. We are presently carrying out similar magnetocaloric measurements for the high quality sample III. There, hopefully, less rounded data will be obtained and analyzed [3] in terms of scaling law equations of state as well as in terms of power laws for special approaches of the critical point; e.g. as a function of temperature for $B_{0}=0$ and as a function of $B_{0}$ for $T=T_{C}$.

\section{REFERENCES}

[1] Bednarz G., Geldart D.J.W., White M.A., Phys.Rev B47 (1993) 14247-14259

[2] Mandelis A. and Zver M.M., J. Appl. Phys. 57 (1985) 4421-4430

[3] Glorieux C., Thoen J., White M.A., Bednarz G. and Geldart D.J.W. (to be published)

[4] Stetter U., Farle M. ,Baberschke K. and Clark W.G., Phys.Rev. B45 (1992) 503-506 